\title{
Work Motivation for Japanese Nursing Assistants in Small- to Medium-Sized Hospitals
}

\author{
Yasushi Kudo, ${ }^{1}$ Shigeri Kido, ${ }^{2}$ Machiko Taruzuka Shahzad, ${ }^{3}$ Emiko Yoshimura, ${ }^{4}$ \\ Akitaka Shibuya $^{1,5}$ and Yoshiharu Aizawa ${ }^{6}$ \\ ${ }^{1}$ Department of Health Care Management, Kitasato University School of Medicine, Sagamihara, Kanagawa, Japan \\ ${ }^{2}$ Department of Fundamentals of Nursing, Kitasato University School of Nursing, Sagamihara, Kanagawa, Japan \\ ${ }^{3}$ Department of Lifespan Development Nursing, Kitasato University School of Nursing, Sagamihara, Kanagawa, \\ Japan \\ ${ }^{4}$ Kawasaki City College of Nursing, Kawasaki, Kanagawa, Japan \\ ${ }^{5}$ Department of Risk Management and Health Care Administration, Kitasato University School of Medicine, \\ Sagamihara, Kanagawa, Japan \\ ${ }^{6}$ Department of Preventive Medicine, Kitasato University School of Medicine, Sagamihara, Kanagawa, Japan
}

\begin{abstract}
Nursing assistants can work without a professional certification to help registered nurses and licensed practical nurses. Nursing assistants engage in various tasks, e.g., washing laundry, cleaning up, and clerk tasks regarding nursing. Enhancing work motivation among nursing assistants is essential for every hospital, because when nursing assistants do their jobs well, it allows registered nurses and licensed practical nurses to complete their own specialized jobs. We examined the predictors significantly associated with nursing assistants' work motivation. For those predictors, we produced items to examine job satisfaction. Those items are classified into intrinsic and extrinsic facets. The subjects for this study were Japanese nursing assistants working in 26 hospitals with 62-376 beds (4 public and 22 private hospitals). A total of 516 nursing assistants were analyzed, with the average age and standard deviation of $42.7 \pm 12.9$ years; the age of 456 female subjects was $43.8 \pm 12.7$ years and that of 60 male subjects was $34.3 \pm 11.0$ years. Our results show that "work motivation" is significantly associated with "free time to do one's own things," "nursing assistants as important partners on the job," "feeling helpful to patients," "participating in decision making," and "job-skill improvement." Free time to do one's own things is an extrinsic item. Hospital administrators must monitor the workload and their quality of life among nursing assistants. All the other significant items are intrinsic. Nursing assistants are not only motivated by money. They highly value the intrinsic nature and experience of their jobs.
\end{abstract}

Keywords: job satisfaction; nursing assistant; professional nurse; questionnaire; work motivation Tohoku J. Exp. Med., 2011, 225 (4), 293-300. (C) 2011 Tohoku University Medical Press

Japanese hospitals employ various professionals: registered nurses, licensed practical nurses, physicians, pharmacists, and certified care workers (Health and Welfare Statistics Association 2010; Ministry of Health, Labour and Welfare 2011a, 2011b). However, they also employ nursing assistants who can work without a professional certification to help registered nurses and licensed practical nurses. Nursing assistants engage in various tasks, e.g., washing laundry, cleaning up, and clerk tasks regarding nursing.

Nursing assistants' tasks may not be considered as prestigious as those of professionals. However, when nursing assistants are well motivated and do good jobs, registered nurses and licensed practical nurses can spend more time doing their own professional jobs. Therefore, research to understand what motivates nursing assistants is essential.
In Japan, there are previous studies such as case reports from individual hospitals regarding nursing assistants (Kobayashi and Yamaguchi 2004; Nabeda and Higuchi 2004; Okubo and Ishizuka 2004; Yamamoto et al. 2004). However, there are no studies that examine predictors of Japanese nursing assistants' work motivation with the cooperation of many hospitals.

When workers' motivation is studied, the focus is most often on job satisfaction (Maslow 1954; Herzberg et al. 1959; McGregor 1960; Robbins 2004). The contents of job satisfaction are generally classified into intrinsic and extrinsic facets. The intrinsic facets (those intrinsic to the nature and experience of working) are a higher order than extrinsic facets. Concrete examples of intrinsic facets are: achievement, recognition, responsibility, decision making, chal-

Received August 4, 2011; revision accepted for publication November 4, 2011. doi: 10.1620/tjem.225.293

Correspondence: Yasushi Kudo, Department of Health Care Management, Kitasato University School of Medicine, 1-15-1 Kitasato, Minami-ku, Sagamihara, Kanagawa 252-0374, Japan.

e-mail: ykudo@med.kitasato-u.ac.jp 
lenging jobs and self-actualization. On the other hand, concrete examples of extrinsic facets are: supervision, salary, working conditions, job security, and security from harm. These two classifications are the most important study concepts in the field of job satisfaction. This concept can be applied to the study of work motivation among nursing assistants.

We examined the associations between work motivation and facets of job satisfaction among nursing assistants by an anonymous self-administered questionnaire survey. The contents of the questionnaire were prepared by classifying the items regarding the nursing assistants' job satisfaction into intrinsic and extrinsic facets.

There has been much research regarding job satisfaction of professional nurses (McClure et al. 1983; Kramer 1990; Leveck and Jones 1996; Scott et al. 1999; Fang 2001; McClure and Hinshaw 2002; Kudo et al. 2010). In the previous studies, these results showed that professional nurses value the intrinsic nature of their jobs. The medical industry exists to protect human life and maintain good health. Therefore, we think that nursing assistants feel they have meaningful jobs in hospitals and are eager to work there. We hypothesize that nursing assistants also feel motivated by fulfilling the intrinsic nature of their jobs.

\section{Subjects and Methods}

\section{Participating hospitals}

In Kanagawa Prefecture, there were 347 hospitals (Ministry of Health, Labour and Welfare 2011b) and an organization of those nursing directors. Workshops are held for those nursing directors in the prefecture a couple of times a year. That organization cooperated fully in this study. Thus, the first author participated in one of those workshops and gave a presentation to enlist cooperation in the present study.

There were 8,739 hospitals in Japan according to survey (Ministry of Health, Labour and Welfare 2011a, 2011b). Among all of these Japanese hospitals, those with fewer than 400 beds made up about $90 \%$. In Kanagawa prefecture, hospitals with fewer than 400 beds also made up about $90 \%$. The Japanese health care system has a high percentage of that sized hospitals, so the hospitals we requested to participate in the present study were of that size. As a result, a total of 26 hospitals with $62-376$ beds ( 4 public and 22 private hospitals) cooperated in the present study.

\section{Range of subjects}

In the nursing department of the hospital, there are workers who are hired to help registered nurses and licensed practical nurses to do their jobs. In the present study, all such workers are called "nursing assistants." Each participating hospital continues to provide nursing education for their nursing assistants, e.g., ethics, human body systems, and patient safety.

The Japanese Ministry of Health, Labour and Welfare investigated the numbers of workers in hospitals in each occupation (Ministry of Health, Labour and Welfare 2011a, 2011b). A total of 31 occupations were surveyed. Among those, health care certifications are required for 26 occupations, e.g., registered nurse, licensed practical nurse, and physician (Japanese Association of Psychiatric Social
Workers 2003; Kojima 2007; Japanese Association of Certified Social Workers 2009; Health and Welfare Statistics Association 2010; Study group of the Certification Exam 2010; Terajima 2011) (see Table 1).

Although there is no longer a certification offered for X-ray technician and health laboratory technician, to obtain certifications for the remaining occupations, it is necessary to receive professional education in training institutes and/or accumulate practical experience to a significant degree, after which, it is necessary to pass an examination. People working in the above 26 occupations are expressed as "professionals" in the present study.

In nursing departments, professionals can work as nursing assistants. However, the present study is focused on the motivation of workers who do not have the certification of health care professionals. In the present study, professionals working as nursing assistants were excluded from the analyses.

For example, in Japan, there is a professional occupation called "certified care worker" (see Table 1). They care for the disabled and elderly who require assistance in their daily lives (Terajima 2011). According to the directors of the nursing department of the participating hospitals in the present study, there were a total of 130 certified care workers serving as nursing assistants. However, certified care workers are professionals; therefore, they were excluded from this study.

On the other hand, in the Japanese health care industry, there are various certifications, other than those for the 26 occupations mentioned above that can only be obtained by receiving training and/

Table 1. Occupations of professional health care providers.

Registered nurse
Licensed practical nurse
Public health nurse
Midwife
Physician
Dentist
Pharmacist
Physical therapist
Occupational therapist
Orthoptist
Speech-Language-Hearing therapist
Prosthetist
Dental hygienist
Dental technician
Radiology technician
X-ray technician
Clinical technologist
Health laboratory technician
Clinical engineering technologist
Massage technician
Judo therapist
Registered dietitian
Dietitian
Psychiatric social worker
Certified social worker
Certified care worker


or by passing a simple exam (Kojima 2007; Study group of the Certification Exam 2010; Aochi 2011; Takano 2011). For example, there is a certification of "home helper" (Takano 2011). Like certified care workers, home helpers care for the disabled and elderly people who require assistance in their daily lives. There are three grades of home helper, from "Grade 1" to "Grade 3", with "Grade 1" being the highest. Even though nursing assistants obtain such certifications, they are not regarded as professionals. Therefore, they were chosen as the subjects in the present study.

\section{Questionnaire contents}

1. Respondents' characteristics

The contents of the respondents' characteristics: age, gender (female/male), employment status (regular/irregular employee), home helper (yes/no), and health care-related certification other than home helper (yes/no).

Before we began this study, the directors of the participating hospitals informed us that many home helpers work as nursing assistants. Therefore, we made the item, "the presence or absence of a home helper certification." If the subjects had a home helper certification, we asked them to specify its grade. Moreover, if subjects had one or more certifications other than that of a home helper certification, they were asked to specify the titles of any health care-related certifications they had.

\section{Work motivation and job satisfaction items}

"Work motivation" was determined by, "I am motivated to work in our hospital." This item is rated on a 7-point scale from "definitely agree" to "definitely disagree."

The 8 items related to job satisfaction are: salary satisfaction, free time to do one's own things, work safety-related instructions, doing jobs that should be done by registered nurses, nursing assistants as important partners on the job, feeling helpful to patients, participating in decision making, and job-skill improvement. The former 4 items are extrinsic facets. The later 4 items are intrinsic facets. These 8 items were all rated on a 7-point scale from "definitely agree" to "definitely disagree."

\section{Participants}

During the period of December 2010 to March 2011, anonymous self-administered questionnaires (original version in Japanese, see the Appendix), with return envelopes and Japanese explanatory literature of the present study, were distributed to 791 nursing assistants. The participants for this study were nursing assistants working in various departments of 26 hospitals with fewer than 400 beds.

As mentioned in the "Range of subjects" section, a total of 130 certified care workers work as nursing assistants in nursing departments in the participating hospitals. However, certified care workers are professionals (see Table 1), so we did not distribute questionnaires to them.

Approximately 1 week after the distribution, the questionnaires were collected in the sealed envelopes that were provided to ensure obtaining truthful answers. The participants could either place the questionnaires in the collection boxes or hand them directly to the persons in charge. The incentive to participate in this survey, as explained in that literature, was to explore new findings that would benefit society.

\section{Respondents}

The respondents' characteristics are shown in Table 1. Of the 791 questionnaires distributed, 608 were collected for a return ratio of $76.9 \%$. The questionnaires that had one or more missing values in each item were excluded from the analyses. We asked each subject to specify if they had one or more health care-related certifications other than that of a home helper certification. If they answered that they had one or more certifications but did not answer what they were, we could not confirm whether the certifications were health-related or not. Therefore, those subjects were excluded from the statistical analyses. One respondent was a certified social worker, and another respondent was a dental hygienist (see Table 1). These two respondents are professionals and were, therefore, excluded from the statistical analyses.

A total of 516 subjects were analyzed. The average age of the analyzed subjects and standard deviation was $42.7 \pm 12.9$ years, while that of 456 women was $43.8 \pm 12.7$ years and that of 60 men was 34.3 \pm 11.0 years.

\section{Statistical Analyses}

To investigate the factors associated with work motivation, standard partial regression coefficients were computed using forcedentry multiple linear regression analysis. Work motivation was the dependent variable. Age, gender (female/male), employment status (regular/irregular employee), health care-related certification (yes/no), salary satisfaction, free time to do one's own things, work safetyrelated instructions, doing jobs that should be done by registered nurses, nursing assistants as important partners on the job, feeling helpful to patients, participating in decision making, and job-skill improvement were the independent variables. Moreover, to examine the relationships between the dependent variable and the independent variables, Pearson's correlation coefficients were calculated and nonparametric tests (the Mann-Whitney $U$ test or the Kruskal-Wallis test) were conducted.

Nursing assistants may have various health-related certifications that can easily be obtained, which greatly differs from certifications given to people with professional occupations. However, efforts to get such certifications are representative of their work motivation. Therefore, we hypothesized that nursing assistants who have such certifications have higher work motivation than those without any certifications. One point was given to the answer, "having a certification of home helper" and/or "having one or more health care-related certifications other than that of a home helper." Zero points were given when a subject had no health care-related certifications.

$\mathrm{PASW}^{\circledR}$ Statistics 18.0 was used for all statistical analyses. The significant level was set at $p<0.05$.

Ethics

The Japanese explanations of the study in addition to the anonymous self-administered questionnaires with return envelopes were distributed to the 791 subjects. The Kitasato University Medical Ethics Committee (B section) approved this study without having to undergo an ethical review committee.

\section{Results}

The distributions of the respondents' characteristics are shown in Table 2. Regarding a certification of home helper, 269 responded that they had a certification of home helper. Among them, there were 11 "Grade 1," 251 "Grade 
Table 2. Distributions of respondents' characteristics.

\begin{tabular}{|c|c|c|}
\hline Characteristic & $\mathrm{N}$ & $\%$ \\
\hline \multicolumn{3}{|l|}{ Age } \\
\hline$\leqq 29$ & 106 & 20.5 \\
\hline $30-39$ & 89 & 17.2 \\
\hline $40-49$ & 143 & 27.7 \\
\hline$\geqq 50$ & 178 & 34.5 \\
\hline \multicolumn{3}{|l|}{ Gender } \\
\hline Female & 456 & 88.4 \\
\hline Male & 60 & 11.6 \\
\hline \multicolumn{3}{|l|}{ Employment status $^{1}$} \\
\hline Regular employee & 262 & 50.8 \\
\hline Irregular employee & 254 & 49.2 \\
\hline \multicolumn{3}{|l|}{ Home helper } \\
\hline Yes & 269 & 52.1 \\
\hline No & 247 & 47.9 \\
\hline \multicolumn{3}{|c|}{ Health care-related certification other than home helper } \\
\hline Yes & 49 & 9.5 \\
\hline No & 467 & 90.5 \\
\hline \multicolumn{3}{|c|}{ Health care-related certification ${ }^{2}$} \\
\hline Yes & 294 & 57.0 \\
\hline No & 222 & 43.0 \\
\hline Total & 516 & 100 \\
\hline
\end{tabular}

${ }^{1}$ Regular employee: a full-time employee who is contracted to work until age of retirement. Irregular employee: an employee other than a regular employee.

2 Yes: Having a certification of home helper and/or one or more health care-related certifications other than that of a home helper. No: Not having any health care-related certifications.
2," 5 "Grade 3," and 2 "Unknown." Regarding health carerelated certifications other than that of a home helper, 49 responded that they had one or more health care-related certifications other than home helper. Some nursing assistants have two or more health care-related certifications. There were certifications related to care giving work, welfare work, medical/dental aid, medical/dental clerkships, and body therapy.

The nonparametric tests are shown in Tables 3 and 4. The Mann-Whitney $U$ tests showed there was a significant difference in the nursing assistants' work motivation with age. The Kruskal-Wallis tests showed there were significant differences in their work motivation with salary satisfaction, free time to do one's own things, work safetyrelated instructions, doing jobs that should be done by registered nurses, nursing assistants as important partners on the job, feeling helpful to patients, participating in decision making, and job-skill improvement.

Pearson's correlation coefficients and the results of multiple regression analysis are also shown in Table 5. "Work motivation" had significant correlation coefficients with salary satisfaction, free time to do one's own things, work safety-related instructions, doing jobs that should be done by registered nurses, nursing assistants as important partners on the job, feeling helpful to patients, participating in decision making, and job-skill improvement. In the results of multiple regression analysis, work motivation was significantly associated with free time to do one's own things, nursing assistants as important partners on the job, feeling helpful to patients, participating in decision making, and job-skill improvement. Variance Inflation Factors were $<2$ in all variables. Adjusted $R^{2}$ value was 0.314 .

Table 3. Distributions of work motivation and characteristics among nursing assistants.

\begin{tabular}{|c|c|c|c|c|c|c|c|c|c|}
\hline Characteristic & $\begin{array}{l}\text { Definitely } \\
\text { agree }\end{array}$ & Agree & $\begin{array}{l}\text { Somewhat } \\
\text { agree }\end{array}$ & $\begin{array}{l}\text { Neither agree } \\
\text { nor disagree }\end{array}$ & $\begin{array}{c}\text { Somewhat } \\
\text { disagree }\end{array}$ & Disagree & $\begin{array}{c}\text { Definitely } \\
\text { disagree }\end{array}$ & Total & $\begin{array}{c}P \\
\text { value }^{1}\end{array}$ \\
\hline \multicolumn{10}{|l|}{$\mathrm{Age}^{2}$} \\
\hline$\leqq 42$ & $21(8.8 \%)$ & $40(16.8 \%)$ & $80(33.6 \%)$ & $62(26.1 \%)$ & $13(5.5 \%)$ & $16(6.7 \%)$ & $6(2.5 \%)$ & $238(100 \%)$ & \\
\hline$\geqq 43$ & $22(7.9 \%)$ & $75(27.0 \%)$ & $99(35.6 \%)$ & $42(15.1 \%)$ & $15(5.4 \%)$ & $18(6.5 \%)$ & $7(2.5 \%)$ & $278(100 \%)$ & 0.022 \\
\hline \multicolumn{10}{|l|}{ Gender } \\
\hline Female & $35(7.7 \%)$ & $102(22.4 \%)$ & $161(35.3 \%)$ & $89(19.5 \%)$ & $26(5.7 \%)$ & $31(6.8 \%)$ & $12(2.6 \%)$ & $456(100 \%)$ & \\
\hline Male & $8(13.3 \%)$ & $13(21.7 \%)$ & $18(30.0 \%)$ & $15(25.0 \%)$ & $2(3.3 \%)$ & $3(5.0 \%)$ & $1(1.7 \%)$ & $60(100 \%)$ & 0.428 \\
\hline \multicolumn{10}{|l|}{ Employment status } \\
\hline Regular employee & $19(7.3 \%)$ & $51(19.5 \%)$ & $92(35.1 \%)$ & $61(23.3 \%)$ & $14(5.3 \%)$ & $16(6.1 \%)$ & $9(3.4 \%)$ & $262(100 \%)$ & \\
\hline Irregular employee & $24(9.4 \%)$ & $64(25.2 \%)$ & $87(34.3 \%)$ & $43(16.9 \%)$ & $14(5.5 \%)$ & $18(7.1 \%)$ & $4(1.6 \%)$ & $254(100 \%)$ & 0.053 \\
\hline \multicolumn{10}{|c|}{ Health care-related certification ${ }^{3}$} \\
\hline Yes & $23(7.8 \%)$ & $62(21.1 \%)$ & $94(32.0 \%)$ & $71(24.1 \%)$ & $13(4.4 \%)$ & $22(7.5 \%)$ & $9(3.1 \%)$ & $294(100 \%)$ & \\
\hline No & $20(9.0 \%)$ & $53(23.9 \%)$ & $85(38.3 \%)$ & $33(14.9 \%)$ & $15(6.8 \%)$ & $12(5.4 \%)$ & $4(1.8 \%)$ & $222(100 \%)$ & 0.069 \\
\hline Total & $43(8.3 \%)$ & $115(22.3 \%)$ & $179(34.7 \%)$ & $104(20.2 \%)$ & $28(5.4 \%)$ & $34(6.6 \%)$ & $13(2.5 \%)$ & $516(100 \%)$ & \\
\hline
\end{tabular}

${ }^{1} P$ values indicate Mann-Whitney $U$ test results.

${ }^{2}$ The average age of the respondents was 42.7 years. Binary categorization was done using an average age of 43 years as a standard.

${ }^{3}$ Yes: Having a certification of home helper and/or one or more health care-related certifications other than that of a home helper. No: Not having any health care-related certifications. 
Table 4. Distributions of work motivation and each variable of job satisfaction among nursing assistants.

\begin{tabular}{|c|c|c|c|c|c|c|c|c|c|}
\hline Variable & Definitely agree & Agree & $\begin{array}{l}\text { Somewhat } \\
\text { agree }\end{array}$ & $\begin{array}{l}\text { Neither agree nor } \\
\text { disagree }\end{array}$ & $\begin{array}{c}\text { Somewhat } \\
\text { disagree }\end{array}$ & Disagree & $\begin{array}{l}\text { Definitely } \\
\text { disagree }\end{array}$ & Total & $P$ value $^{1}$ \\
\hline \multicolumn{10}{|l|}{ Salary satisfaction } \\
\hline Definitely agree & $6(46.2 \%)$ & $2(15.4 \%)$ & $3(23.1 \%)$ & $0(0.0 \%)$ & $0(0.0 \%)$ & $1(7.7 \%)$ & $1(7.7 \%)$ & $13(100 \%)$ & \\
\hline Agree & $4(12.9 \%)$ & $17(54.8 \%)$ & $7(22.6 \%)$ & $2(6.5 \%)$ & $1(3.2 \%)$ & $0(0.0 \%)$ & $0(0.0 \%)$ & $31(100 \%)$ & \\
\hline Somewhat agree & $5(12.5 \%)$ & $9(22.5 \%)$ & $19(47.5 \%)$ & $5(12.5 \%)$ & $2(5.0 \%)$ & $0(0.0 \%)$ & $0(0.0 \%)$ & $40(100 \%)$ & \\
\hline Neither agree nor disagree & $4(4.8 \%)$ & $12(14.3 \%)$ & $35(41.7 \%)$ & $26(31.0 \%)$ & $5(6.0 \%)$ & $2(2.4 \%)$ & $0(0.0 \%)$ & $84(100 \%)$ & \\
\hline Somewhat disagree & $8(7.2 \%)$ & $22(19.8 \%)$ & $38(34.2 \%)$ & $28(25.2 \%)$ & $7(6.3 \%)$ & $5(4.5 \%)$ & $3(2.7 \%)$ & $111(100 \%)$ & \\
\hline Disagree & $7(6.1 \%)$ & $29(25.4 \%)$ & $44(38.6 \%)$ & $19(16.7 \%)$ & $4(3.5 \%)$ & $9(7.9 \%)$ & $2(1.8 \%)$ & $114(100 \%)$ & \\
\hline Definitely disagree & $9(7.3 \%)$ & $24(19.5 \%)$ & $33(26.8 \%)$ & $24(19.5 \%)$ & $9(7.3 \%)$ & $17(13.8 \%)$ & $7(5.7 \%)$ & $123(100 \%)$ & $<0.001$ \\
\hline \multicolumn{10}{|l|}{ Free time to do one's own things } \\
\hline Definitely agree & $12(34.3 \%)$ & $12(34.3 \%)$ & $8(22.9 \%)$ & $2(5.7 \%)$ & $0(0.0 \%)$ & $1(2.9 \%)$ & $0(0.0 \%)$ & $35(100 \%)$ & \\
\hline Agree & $9(12.0 \%)$ & $23(30.7 \%)$ & $31(41.3 \%)$ & $6(8.0 \%)$ & $3(4.0 \%)$ & $3(4.0 \%)$ & $0(0.0 \%)$ & $75(100 \%)$ & \\
\hline Somewhat agree & $6(4.4 \%)$ & $31(22.6 \%)$ & $57(41.6 \%)$ & $30(21.9 \%)$ & $5(3.6 \%)$ & $6(4.4 \%)$ & $2(1.5 \%)$ & $137(100 \%)$ & \\
\hline Neither agree nor disagree & $5(6.1 \%)$ & $12(14.6 \%)$ & $29(35.4 \%)$ & $20(24.4 \%)$ & $6(7.3 \%)$ & $6(7.3 \%)$ & $4(4.9 \%)$ & $82(100 \%)$ & \\
\hline Somewhat disagree & $2(2.7 \%)$ & $16(21.3 \%)$ & $27(36.0 \%)$ & $19(25.3 \%)$ & $6(8.0 \%)$ & $4(5.3 \%)$ & $1(1.3 \%)$ & $75(100 \%)$ & \\
\hline Disagree & $5(6.8 \%)$ & $16(21.9 \%)$ & $17(23.3 \%)$ & $16(21.9 \%)$ & $6(8.2 \%)$ & $9(12.3 \%)$ & $4(5.5 \%)$ & $73(100 \%)$ & \\
\hline Definitely disagree & $4(10.3 \%)$ & $5(12.8 \%)$ & $10(25.6 \%)$ & $11(28.2 \%)$ & $2(5.1 \%)$ & $5(12.8 \%)$ & $2(5.1 \%)$ & $39(100 \%)$ & $<0.001$ \\
\hline \multicolumn{10}{|l|}{ Work safety-related instructions } \\
\hline Definitely agree & $9(47.4 \%)$ & $6(31.6 \%)$ & $3(15.8 \%)$ & $0(0.0 \%)$ & $0(0.0 \%)$ & $1(5.3 \%)$ & $0(0.0 \%)$ & $19(100 \%)$ & \\
\hline Agree & $7(11.5 \%)$ & $23(37.7 \%)$ & $20(32.8 \%)$ & $5(8.2 \%)$ & $3(4.9 \%)$ & $2(3.3 \%)$ & $1(1.6 \%)$ & $61(100 \%)$ & \\
\hline Somewhat agree & $8(7.4 \%)$ & $29(26.9 \%)$ & $43(39.8 \%)$ & $24(22.2 \%)$ & $3(2.8 \%)$ & $1(0.9 \%)$ & $0(0.0 \%)$ & $108(100 \%)$ & \\
\hline Neither agree nor disagree & $7(5.9 \%)$ & $27(22.7 \%)$ & $41(34.5 \%)$ & $34(28.6 \%)$ & $4(3.4 \%)$ & $5(4.2 \%)$ & $1(0.8 \%)$ & $119(100 \%)$ & \\
\hline Somewhat disagree & $7(7.5 \%)$ & $11(11.8 \%)$ & $37(39.8 \%)$ & $21(22.6 \%)$ & $7(7.5 \%)$ & $9(9.7 \%)$ & $1(1.1 \%)$ & $93(100 \%)$ & \\
\hline Disagree & $0(0.0 \%)$ & $12(16.7 \%)$ & $28(38.9 \%)$ & $14(19.4 \%)$ & $6(8.3 \%)$ & $11(15.3 \%)$ & $1(1.4 \%)$ & $72(100 \%)$ & \\
\hline Definitely disagree & $5(11.4 \%)$ & $7(15.9 \%)$ & $7(15.9 \%)$ & $6(13.6 \%)$ & $5(11.4 \%)$ & $5(11.4 \%)$ & $9(20.5 \%)$ & $44(100 \%)$ & $<0.001$ \\
\hline \multicolumn{10}{|c|}{ Doing jobs that should be done by registered nurses } \\
\hline Definitely agree & $1(3.1 \%)$ & $6(18.8 \%)$ & $8(25.0 \%)$ & $5(15.6 \%)$ & $1(3.1 \%)$ & $4(12.5 \%)$ & $7(21.9 \%)$ & $32(100 \%)$ & \\
\hline Agree & $7(15.2 \%)$ & $10(21.7 \%)$ & $14(30.4 \%)$ & $6(13.0 \%)$ & $2(4.3 \%)$ & $6(13.0 \%)$ & $1(2.2 \%)$ & $46(100 \%)$ & \\
\hline Somewhat agree & $9(6.1 \%)$ & $32(21.6 \%)$ & $54(36.5 \%)$ & $31(20.9 \%)$ & $12(8.1 \%)$ & $9(6.1 \%)$ & $1(0.7 \%)$ & $148(100 \%)$ & \\
\hline Neither agree nor disagree & $4(3.9 \%)$ & $12(11.8 \%)$ & $43(42.2 \%)$ & $30(29.4 \%)$ & $6(5.9 \%)$ & $6(5.9 \%)$ & $1(1.0 \%)$ & $102(100 \%)$ & \\
\hline Somewhat disagree & $2(5.3 \%)$ & $7(18.4 \%)$ & $14(36.8 \%)$ & $8(21.1 \%)$ & $3(7.9 \%)$ & $4(10.5 \%)$ & $0(0.0 \%)$ & $38(100 \%)$ & \\
\hline Disagree & $12(12.9 \%)$ & $31(33.3 \%)$ & $29(31.2 \%)$ & $16(17.2 \%)$ & $1(1.1 \%)$ & $4(4.3 \%)$ & $0(0.0 \%)$ & $93(100 \%)$ & \\
\hline Definitely disagree & $8(14.0 \%)$ & $17(29.8 \%)$ & $17(29.8 \%)$ & $8(14.0 \%)$ & $3(5.3 \%)$ & $1(1.8 \%)$ & $3(5.3 \%)$ & $57(100 \%)$ & $<0.001$ \\
\hline \multicolumn{10}{|c|}{ Nursing assistants as important partners on the job } \\
\hline Definitely agree & $13(39.4 \%)$ & $6(18.2 \%)$ & $7(21.2 \%)$ & $5(15.2 \%)$ & $1(3.0 \%)$ & $1(3.0 \%)$ & $0(0.0 \%)$ & $33(100 \%)$ & \\
\hline Agree & $13(14.1 \%)$ & $29(31.5 \%)$ & $32(34.8 \%)$ & $15(16.3 \%)$ & $2(2.2 \%)$ & $1(1.1 \%)$ & $0(0.0 \%)$ & $92(100 \%)$ & \\
\hline Somewhat agree & $4(3.1 \%)$ & $39(30.2 \%)$ & $59(45.7 \%)$ & $12(9.3 \%)$ & $9(7.0 \%)$ & $3(2.3 \%)$ & $3(2.3 \%)$ & $129(100 \%)$ & \\
\hline Neither agree nor disagree & $7(5.8 \%)$ & $16(13.3 \%)$ & $36(30.0 \%)$ & $43(35.8 \%)$ & $9(7.5 \%)$ & $9(7.5 \%)$ & $0(0.0 \%)$ & $120(100 \%)$ & \\
\hline Somewhat disagree & $1(1.4 \%)$ & $9(12.9 \%)$ & $27(38.6 \%)$ & $21(30.0 \%)$ & $5(7.1 \%)$ & $6(8.6 \%)$ & $1(1.4 \%)$ & $70(100 \%)$ & \\
\hline Disagree & $2(4.5 \%)$ & $9(20.5 \%)$ & $16(36.4 \%)$ & $5(11.4 \%)$ & $1(2.3 \%)$ & $8(18.2 \%)$ & $3(6.8 \%)$ & $44(100 \%)$ & \\
\hline Definitely disagree & $3(10.7 \%)$ & $7(25.0 \%)$ & $2(7.1 \%)$ & $3(10.7 \%)$ & $1(3.6 \%)$ & $6(21.4 \%)$ & $6(21.4 \%)$ & $28(100 \%)$ & $<0.001$ \\
\hline \multicolumn{10}{|l|}{ Feeling helpful to patients } \\
\hline Definitely agree & $18(46.2 \%)$ & $10(25.6 \%)$ & $7(17.9 \%)$ & $1(2.6 \%)$ & $1(2.6 \%)$ & $2(5.1 \%)$ & $0(0.0 \%)$ & $39(100 \%)$ & \\
\hline Agree & $15(13.0 \%)$ & $41(35.7 \%)$ & $30(26.1 \%)$ & $11(9.6 \%)$ & $6(5.2 \%)$ & $9(7.8 \%)$ & $3(2.6 \%)$ & $115(100 \%)$ & \\
\hline Somewhat agree & $5(2.1 \%)$ & $53(22.1 \%)$ & $96(40.0 \%)$ & $59(24.6 \%)$ & $11(4.6 \%)$ & $12(5.0 \%)$ & $4(1.7 \%)$ & $240(100 \%)$ & \\
\hline Neither agree nor disagree & $4(4.4 \%)$ & $7(7.8 \%)$ & $35(38.9 \%)$ & $31(34.4 \%)$ & $5(5.6 \%)$ & $7(7.8 \%)$ & $1(1.1 \%)$ & $90(100 \%)$ & \\
\hline Somewhat disagree & $0(0.0 \%)$ & $1(5.9 \%)$ & $7(41.2 \%)$ & $2(11.8 \%)$ & $4(23.5 \%)$ & $2(11.8 \%)$ & $1(5.9 \%)$ & $17(100 \%)$ & \\
\hline Disagree & $0(0.0 \%)$ & $3(30.0 \%)$ & $4(40.0 \%)$ & $0(0.0 \%)$ & $1(10.0 \%)$ & $1(10.0 \%)$ & $1(10.0 \%)$ & $10(100 \%)$ & \\
\hline Definitely disagree & $1(20.0 \%)$ & $0(0.0 \%)$ & $0(0.0 \%)$ & $0(0.0 \%)$ & $0(0.0 \%)$ & $1(20.0 \%)$ & $3(60.0 \%)$ & $5(100 \%)$ & $<0.001$ \\
\hline \multicolumn{10}{|l|}{ Participating in decision making } \\
\hline Definitely agree & $8(40.0 \%)$ & $8(40.0 \%)$ & $3(15.0 \%)$ & $1(5.0 \%)$ & $0(0.0 \%)$ & $0(0.0 \%)$ & $0(0.0 \%)$ & $20(100 \%)$ & \\
\hline Agree & $10(18.5 \%)$ & $22(40.7 \%)$ & $10(18.5 \%)$ & $11(20.4 \%)$ & $1(1.9 \%)$ & $0(0.0 \%)$ & $0(0.0 \%)$ & $54(100 \%)$ & \\
\hline Somewhat agree & $7(4.6 \%)$ & $40(26.3 \%)$ & $68(44.7 \%)$ & $26(17.1 \%)$ & $6(3.9 \%)$ & $5(3.3 \%)$ & $0(0.0 \%)$ & $152(100 \%)$ & \\
\hline Neither agree nor disagree & $11(8.9 \%)$ & $16(12.9 \%)$ & $49(39.5 \%)$ & $33(26.6 \%)$ & $8(6.5 \%)$ & $6(4.8 \%)$ & $1(0.8 \%)$ & $124(100 \%)$ & \\
\hline Somewhat disagree & $2(4.8 \%)$ & $6(14.3 \%)$ & $17(40.5 \%)$ & $7(16.7 \%)$ & $4(9.5 \%)$ & $6(14.3 \%)$ & $0(0.0 \%)$ & $42(100 \%)$ & \\
\hline Disagree & $3(3.8 \%)$ & $16(20.5 \%)$ & $22(28.2 \%)$ & $18(23.1 \%)$ & $7(9.0 \%)$ & $7(9.0 \%)$ & $5(6.4 \%)$ & $78(100 \%)$ & \\
\hline Definitely disagree & $2(4.3 \%)$ & $7(15.2 \%)$ & $10(21.7 \%)$ & $8(17.4 \%)$ & $2(4.3 \%)$ & $10(21.7 \%)$ & $7(15.2 \%)$ & $46(100 \%)$ & $<0.001$ \\
\hline \multicolumn{10}{|l|}{ Job-skill improvement } \\
\hline Definitely agree & $10(47.6 \%)$ & $7(33.3 \%)$ & $3(14.3 \%)$ & $0(0.0 \%)$ & $0(0.0 \%)$ & $1(4.8 \%)$ & $0(0.0 \%)$ & $21(100 \%)$ & \\
\hline Agree & $7(11.5 \%)$ & $34(55.7 \%)$ & $17(27.9 \%)$ & $2(3.3 \%)$ & $0(0.0 \%)$ & $1(1.6 \%)$ & $0(0.0 \%)$ & $61(100 \%)$ & \\
\hline Somewhat agree & $9(5.7 \%)$ & $44(27.7 \%)$ & $76(47.8 \%)$ & $20(12.6 \%)$ & $4(2.5 \%)$ & $6(3.8 \%)$ & $0(0.0 \%)$ & $159(100 \%)$ & \\
\hline Neither agree nor disagree & $11(7.8 \%)$ & $11(7.8 \%)$ & $50(35.5 \%)$ & $55(39.0 \%)$ & $7(5.0 \%)$ & $6(4.3 \%)$ & $1(0.7 \%)$ & $141(100 \%)$ & \\
\hline Somewhat disagree & $2(3.8 \%)$ & $9(17.3 \%)$ & $21(40.4 \%)$ & $12(23.1 \%)$ & $4(7.7 \%)$ & $4(7.7 \%)$ & $0(0.0 \%)$ & $52(100 \%)$ & \\
\hline Disagree & $1(1.9 \%)$ & $6(11.5 \%)$ & $10(19.2 \%)$ & $14(26.9 \%)$ & $8(15.4 \%)$ & $10(19.2 \%)$ & $3(5.8 \%)$ & $52(100 \%)$ & \\
\hline Definitely disagree & $3(10.0 \%)$ & $4(13.3 \%)$ & $2(6.7 \%)$ & $1(3.3 \%)$ & $5(16.7 \%)$ & $6(20.0 \%)$ & $9(30.0 \%)$ & $30(100 \%)$ & $<0.001$ \\
\hline Total & $43(8.3 \%)$ & $115(22.3 \%)$ & $179(34.7 \%)$ & $104(20.2 \%)$ & $28(5.4 \%)$ & $34(6.6 \%)$ & $13(2.5 \%)$ & $516(100 \%)$ & \\
\hline
\end{tabular}

${ }^{1} P$ values indicate Kruskal-Wallis test results. 
Table 5. Predictors associated with nursing assistants' work motivation rated on a 7-point scale from definitely agree to definitely disagree.

\begin{tabular}{lrrrrr}
\hline \multicolumn{1}{c}{ Variable } & $\mathrm{r}^{1}$ & $P$ value & $\beta^{2}$ & $P$ value & $\mathrm{VIF}^{3}$ \\
\hline Age & 0.038 & 0.390 & 0.040 & 0.303 & 1.152 \\
Gender (female/male) & -0.044 & 0.316 & 0.006 & 0.870 & 0.126 \\
Employment status (regular/irregular employee) & -0.074 & 0.091 & -0.043 & 0.273 & 0.482 \\
Health care-related certification (yes/no) & -0.075 & 0.088 & -0.027 & 0.762 \\
Salary satisfaction & 0.222 & $<0.001$ & 0.013 & 0.035 \\
Free time to do one's own things & 0.260 & $<0.001$ & 0.088 & 0.728 \\
Work safety-related instructions & 0.338 & $<0.001$ & 0.017 & 1.089 \\
Doing jobs that should be done by registered nurses & -0.185 & $<0.001$ & -0.009 & 0.825 \\
Nursing assistants as important partners on the job & 0.333 & $<0.001$ & 0.106 & 0.016 \\
Feeling helpful to patients & 0.319 & $<0.001$ & 0.167 & $<0.001$ \\
Participating in decision making & 0.373 & $<0.001$ & 0.132 & 0.004 \\
Job-skill improvement & 0.485 & $<0.001$ & 0.314 & $<0.001$ \\
\end{tabular}

Adjusted $R^{2}=0.314$

${ }^{1} \mathrm{r}$, Pearson's correlation coefficient

${ }^{2} \beta$, standardized partial regression coefficient

${ }^{3}$ VIF, variance inflation factor

${ }^{4}$ Yes: Having a certification of home helper and/or one or more health care-related certifications other than that of a home helper. No: Not having any health care-related certifications.

Note: To conduct multiple regression analysis, the following system was used. Work motivation was the dependent variable. Age, gender, employment status, health care-related certification, salary satisfaction, free time to do one's own things, work safety-related instructions, doing jobs that should be done by registered nurses, nursing assistants as important partners on the job, feeling helpful to patients, participating in decision making, and job skill-improvement were the independent variables, all of which were fixed in this model. Concerning work motivation, 1 to 7 points were given in the order from definitely disagree to definitely agree. Concerning age, the actual age was used as a continuous variable. The coding of dichotomous variables was: gender (female $=1$, male $=0$ ); employment status (regular employee $=1$, irregular employee $=0$ ); and health care-related certification (yes $=1$, no $=0$ ). Concerning salary satisfaction, free time to do one's own things, work safety-related instructions, doing jobs that should be done by registered nurses, nursing assistants as important partners on the job, feeling helpful to patients, participating in decision making, and job-skill improvement, 1 to 7 points were given in the order from definitely disagree to definitely agree. To calculate the Pearson's correlation coefficients, we also made the same coding of points of each variable.

\section{Discussion}

By focusing on job satisfaction, we discussed the predictors related to the work motivation of nursing assistants. In the previous studies of job satisfaction (Maslow 1954; Herzberg et al. 1959; McGregor 1960; Robbins 2004), the contents of job satisfaction are generally classified into intrinsic and extrinsic facets. This concept was adopted for the present study, as well, and a questionnaire was produced based on that. As a result, nursing assistants as important partners on the job, feeling helpful to patients, participating in decision making, and job-skill improvement were significant variables, which are intrinsic facets. Free time to do one's own things was a significant variable among extrinsic facets.

The concepts in the "magnet hospital" studies are especially well known (McClure et al. 1983; Kramer 1990; Scott et al. 1999; McClure and Hinshaw 2002). These studies were conducted during the national nursing shortage in the 1980s in the United States. To remedy this shortage, researchers studied the characteristics of the hospitals where professional nurses felt that their jobs were meaningful and maintained their high job retention rates. The researchers selected model hospitals from among the hospitals in the United States, and studied their common characteristics by conducting an interview survey. As a result, the specialties of "magnet hospitals" were discovered. Those hospitals develop a working environment that satisfies intrinsic facets (e.g., autonomy and professional development, such as continuing education, formal education, and career development).

In other previous studies, other than the "magnet hospital" studies, similar results were obtained (Leveck and Jones 1996; Fang 2001; Kudo et al. 2010). Leveck and Jones (1996) reported that "professional job satisfaction" is one of the predictors of retention. Fang (2001) also reported that "turnover cognition," which refers to thinking about quitting one's job, was significantly associated with "professional commitment." In our previous study (Kudo et al. 2010), "nurses' dedication to patients" and "nurses' contribution to their hospitals" were significantly associated with "work as specialists." These results imply that nurses truly value the intrinsic nature of their jobs.

The present study is focused on nursing assistants. As a result, their work motivation was significantly associated with all variables regarding intrinsic facets. The people 
working in the medical industry help protect human life and maintain good health. Although nursing assistants have not received professional nursing education, they feel their jobs of helping to protect people's lives and health are extremely important. Nursing assistants cannot be motivated to work simply by controlling their salary levels. Hospital administrators and professional nurses must consider these new findings presented in this study.

Work motivation was significantly associated with free time to do one's own things. To do a good job, it is essential to have adequate free time. Moreover, in Japan, it is important to realize a good balance between "human labor" and "quality of life" (Kishi 2009). Each worker is a human being, not just a cog in the wheel. Hospital administrators must monitor the amount of work their nursing assistants do, while, at the same time, consider their quality of life. In the present study, there were no questions about nursing assistants' overtime hours, irregular shifts, or opinions regarding their quality of life. These issues warrant further study.

We did not distribute questionnaires to certified care workers because they are professionals. According to the directors of the nursing department of the 26 hospitals in the present study, there were a total of 130 certified care workers serving as nursing assistants. In Japan, the shortage of nurses has become serious (Sawada 1997; Takahashi 2008). In these circumstances, the employment of certified care workers may become more useful. It is also necessary to investigate their working environments. This issue warrants further study.

In Japan, there are many health care-related certifications that can be obtained relatively easily (Kojima 2007; Study group of the certification exam 2010; Aochi 2011; Takano 2011). The results of this study indicate that nursing assistants obtained such certifications voluntarily. These certifications are evaluated differently among hospitals. However, it may be useful to use these certifications for developing the abilities of nursing assistants. In the future, we plan to investigate the opinions of health care providers.

There have been studies regarding latent factors of Japanese professional nurses' job satisfaction using factor analysis (Shimazu 1994; Sawada et al. 2002; Kudo et al. 2010). However, to our knowledge, there have been no studies on latent factors of job satisfaction among Japanese nursing assistants. If these latent factors are identified, they could be useful tools to apply to the study of work motivation and job retention. In future studies, we will investigate the latent factors among nursing assistants using factor analysis.

The present study is focused on job satisfaction regarding nursing assistants' work motivation. However, it is also necessary to consider various things such as health conditions and stress as predictors related to work motivation. In future studies, we will also study nursing assistants' work motivation from other viewpoints besides job satisfaction.

\section{Limitations}

The first limitation is that because a cross-sectional design was used, it is difficult to identify causal relations. The second limitation is that nursing assistants' opinions in hospitals that did not participate in the present study were not analyzed. The present study focuses on only Kanagawa prefectural hospitals. Therefore, the generalizability of our results is limited. It is necessary to investigate the hospitals in other prefectures.

\section{Conclusions}

"Work motivation" is significantly associated with "free time to do one's own things," "nursing assistants as important partners on the job," "feeling helpful to patients," "participating in decision making," and "job-skill improvement." To acquire enough free time, it is necessary that hospital administrators pay close attention to their nursing assistants' workloads. The other significant variables, other than free time, are all of an intrinsic nature. The people working in the medical industry help protect human life and maintain good health. Nursing assistants take pride in their work in this industry. Nursing assistants are not only motivated by money. They highly value the intrinsic nature and experience of their jobs.

\section{Acknowledgments}

This study was supported by a Kitasato University Research Grant for Young Researchers.

\section{Conflict of Interest}

The authors declare no conflicts of interest.

\section{References}

Aochi, K. (2011) A book to completely understand medical clerkship, Nihonbungeisha, Tokyo (in Japanese).

Fang, Y. (2001) Turnover propensity and its causes among Singapore nurses: an empirical study. Int. J. Hum. Resour. Manage., $12,859-871$.

Health and Welfare Statistics Association. (2010) Health Care Provision. In Annual Statistical Report of National Health Conditions 2010/2011, Tokyo pp. 170-212 (in Japanese).

Herzberg, F., Mausner, B. \& Snyderman, B.B. (1959) The Motivation to Work, Wiley, New York, NY.

Japanese Association of Certified Social Workers (2009) Complete guide of certified social workers, 3rd ed., Mineruva Bookstore, Kyoto (in Japanese).

Japanese Association of Psychiatric Social Workers (2003) Complete guide of psychiatric social workers, Mineruva Bookstore, Kyoto (in Japanese).

Kishi, R. (2009) Harmonization of human labor and quality of life: Thinking on a new system for working. The Institute for science of labor, Kawasaki (in Japanese).

Kobayashi, H. \& Yamaguchi, E. (2004) The tasks and education and training among nursing assistants considering patients' care environment. Nurs. Mang., 6, 10-17 (in Japanese).

Kojima, M. (2007) A work guide for care, welfare, and medical treatment. Nippon Jitsugyo Shuppan, Tokyo (in Japanese).

Kramer, M. (1990) The magnet hospitals. Excellence revisited. $J$. Nurs. Adm. 20, 35-44.

Kudo, Y., Kido, S., Shahzad, M.T., Shida, K., Satoh, T. \& Aizawa, 
Y. (2010) Enhancing work motivation for Japanese female nurses in small to medium-sized private hospitals by analyzing job satisfaction. Tohoku J. Exp. Med., 220, 237-245.

Leveck, M.L. \& Jones, C.B. (1996) The nursing practice environment, staff retention, and quality of care. Res. Nurs. Health, 19, 331-343.

Maslow, A. (1954) Motivation and Personality. Harper \& Row, New York, NY.

McClure, M.L. \& Hinshaw, A.S. (2002) Magnet Hospitals Revisited: Attraction and Retention of Professional Nurses. American Nurses Association, Washington, D.C.

McClure, M.L., Poulin, M.A., Sovie, M.D. \& Wandelt, M.A. (1983) Magnet Hospitals: Attraction and Retention of Professional Nurses. American Nurses' Association, Kansas City, MO.

McGregor, D. (1960) The Human Side of Enterprise. McGrawHill, New York, NY

Ministry of Health, Labour and Welfare (2011a) Survey of Medical Institutions \& Hospital Report (Volume 1: National Tables) 12009. Health and Welfare Statistics Association, Tokyo (in Japanese).

Ministry of Health, Labour and Welfare (2011b) Survey of Medical Institutions \& Hospital Report (Volume 2: Prefectural Tables) /2009. Health and Welfare Statistics Association, Tokyo (in Japanese).

Nabeda, A. \& Higuchi, H. (2004) Economic effectiveness working with nursing assistants: cost down by doing away with primary disinfection. Nurs. Mang., 6, 31-36 (in Japanese).

Okubo, T. \& Ishizuka, A. (2004) Strategic employment and education in-service training among nursing assistants: introducing a temp staffer. Nurs. Mang., 6, 25-30 (in Japanese).

Robbins, S.P. (2004) Basic Motivation Concepts. In Essentials of Organizational Behavior, 8th ed., Prentice Hall, Upper Saddle River, NJ, pp. 47-62.

Sawada, A. (1997) The nurse shortage problem in Japan. Nurs. Ethics., 4, 245-252.

Sawada, T., Hadano, H. \& Sakai, J. (2002) Job satisfaction of nurses: a covariance structure analysis of the job satisfaction and its determinants. Bull. Ehime Col. Health Sci., 15, 1-9 (in Japanese).

Scott, J.G., Sochalski, J. \& Aiken, L. (1999) Review of magnet hospital research: findings and implications for professional nursing practice. J. Nurs. Adm., 29, 9-19.

Shimazu, N. (1994) The structure of internal-marketing of medical institution: using an investigation of job satisfaction of nurses. Byouin Kanri, 31, 239-247 (in Japanese).

Study group of the certification exam (2010) Book to Understand the Work of Welfare and Care, and the Certification, Jitsumukyoiku Shuppan, Tokyo (in Japanese).

Takahashi, Y. (2008) Toward the construction of a local network system to train and develop new nurses: state and matter about shortage of nurses in rural areas. Study Adult Edu., 26, 25-42 (in Japanese)

Takano, K. (2011) Home Helper Handbook, Shinsei Publishing, Tokyo (in Japanese).
Terajima, A. (2011) A Book for People Aspiring to Become Certified Care Workers, Seibido Shuppan, Tokyo (in Japanese).

Yamamoto, A., Takahashi, M., Ogawa, Y. \& Kawakatsu, M. (2004) Independent organization among nursing assistants: selfsustaining nursing assistants supporting professional. Nurs. Mang., 6, 18-24 (in Japanese).

\section{Appendix}

Questionnaire items (original version in Japanese)

\section{Job satisfaction}

"Salary satisfaction"

1. I am satisfied with my present salary level in our hospital.

"Free time to do one's own things"

2. Although I work in our hospital, I have free time to do my own things after work.

\section{"Work safety-related instructions"}

3. In our hospital, nursing assistants receive instructions so as not to endanger themselves.

"Doing jobs that should be done by registered nurses"

4. The registered nurses in our hospital, without thinking carefully about it, ask nursing assistants to do jobs that should be done by registered nurses.

"Nursing assistants as important partners on the job"

5. The registered nurses in our hospital recognize nursing assistants as important partners on the job.

"Feeling helpful to patients"

6. Working in our hospital, I feel I am helpful to patients.

"Participating in decision making"

7. In our hospital, nursing assistants have opportunities to participate in decision making.

"Job-skill improvement"

8. Job skills can be improved in our hospital.

\section{Work motivation}

9. I am motivated to work in our hospital. 